\title{
Effects of Fasting on ${ }^{18}$ F-DCFPyL Uptake in Prostate Cancer Lesions and Tissues with Known High Physiologic Uptake
}

\author{
Maurits Wondergem ${ }^{1}$, Friso M. van der Zant ${ }^{1}$, Peter W. Vlottes ${ }^{2}$, and Remco J.J. Knol ${ }^{1}$ \\ ${ }^{1}$ Department of Nuclear Medicine, Noordwest Ziekenhuisgroep, Alkmaar, The Netherlands; and ${ }^{2}$ Radiopharmacy, Noordwest \\ Ziekenhuisgroep, Alkmaar, The Netherlands
}

\begin{abstract}
In the literature, a 4- to $6-\mathrm{h}$ fast is recommended before a patient undergoes PET/CT with 2-(3-(1-carboxy-5-[(6-18F-fluoro-pyridine-3carbonyl)-amino]-pentyl)-ureido)-pentanedioic acid ( $\left.{ }^{18} \mathrm{~F}-\mathrm{DCFPyL}\right)$; however, a scientific underpinning for this recommendation is lacking. Therefore, we performed a study to determine the impact of fasting on ${ }^{18} \mathrm{~F}-\mathrm{DCFPyL}$ uptake. Methods: The study included 50 patients who fasted at least $6 \mathrm{~h}$ before ${ }^{18} \mathrm{~F}$-DCFPyL administration and 50 patients who did not. Activity $\left(S U V_{\max }\right)$ was measured in lesions characteristic of prostate cancer and in normal tissues known to express high physiologic uptake. Results: Uptake in suspected lesions did not differ between the cohorts. ${ }^{18} \mathrm{~F}-\mathrm{DCFPyL}$ uptake in the submandibular gland, liver, and spleen was significantly higher in the fasting than the nonfasting cohort. Conclusion: Our data show that fasting does not significantly affect ${ }^{18} \mathrm{~F}-\mathrm{DCFPyL}$ uptake in suspected malignant lesions but does result in significantly lower ${ }^{18} \mathrm{~F}-\mathrm{DCFPyL}$ uptake in tissues with high physiologic uptake. The absolute differences in uptake were relatively small; therefore, the effects of fasting on the diagnostic performance can be considered negligible.
\end{abstract}

Key Words: genitourinary; acquisition protocol; ${ }^{18} \mathrm{~F}-\mathrm{DCFPyL}$; PET/ CT; PSMA; prostate cancer

J Nucl Med 2018; 59:1081-1084

DOI: 10.2967/jnumed.117.207316

\section{$\mathbf{P}$} rostate-specific membrane antigen (PSMA)-targeted PET imaging is being increasingly applied in prostate cancer. In patients with a slightly elevated prostate-specific antigen level, PSMA-targeted PET/CT may detect and localize prostate cancer lesions that would not be detected by CT, MRI, or ${ }^{18} \mathrm{~F}$-choline PET/CT (1). One promising ${ }^{18} \mathrm{~F}$-labeled PSMA ligand is 2-(3-(1carboxy-5-[(6-18 F-fluoro-pyridine-3-carbonyl)-amino]-pentyl)-ureido)pentanedioic acid $\left({ }^{18} \mathrm{~F}-\mathrm{DCFPyL}\right)(2)$. In the literature, fasting for between 4 and $6 \mathrm{~h}$ before a patient undergoes ${ }^{18} \mathrm{~F}$-DCFPyL PET/ CT is frequently recommended (3). The joint guideline on ${ }^{68} \mathrm{Ga}-$ PSMA PET/CT by the European Association of Nuclear Medicine and the Society of Nuclear Medicine and Molecular Imaging gives no specific guidance on patient preparation (4), and no guideline on ${ }^{18} \mathrm{~F}$-labeled PSMA radiopharmaceuticals has yet been established. To the best of our knowledge, there is no evidence supporting

Received Dec. 20, 2017; revision accepted Feb. 6, 2018.

For correspondence or reprints contact: Maurits Wondergem, Noordwest

Ziekenhuisgroep, Wilhelminalaan 12, 1815 JD, 0030, The Netherlands

E-mail: m.wondergem@nwz.nl

Published online Mar. 1, 2018.

COPYRIGHT (C 2018 by the Society of Nuclear Medicine and Molecular Imaging. fasting before ${ }^{18} \mathrm{~F}-\mathrm{DCFPyL}$ PET/CT. Therefore, this study aimed to determine the impact of fasting on ${ }^{18} \mathrm{~F}-\mathrm{DCFPyL}$ uptake in organs with known high physiologic uptake and in lesions characteristic of prostate cancer metastases.

\section{MATERIALS AND METHODS}

\section{Patients}

Starting in November 2016, the data of all patients who underwent ${ }^{18} \mathrm{~F}-\mathrm{DCFPyL} \mathrm{PET} / \mathrm{CT}$ were prospectively entered into a database. Initially, patients fasted at least $6 \mathrm{~h}$ before ${ }^{18} \mathrm{~F}-\mathrm{DCFPyL}$ administration. After evaluation of the acquisition protocol, fasting was discarded because evidence of its necessity was lacking in the literature. A consecutive 50 patients who fasted and 50 who did not were included in the study. ${ }^{18} \mathrm{~F}-\mathrm{DCFPyL}$ PET/CT was performed for primary staging, for evaluation of biochemical recurrence, or for measurement of response to systemic therapy. Any follow-up scans of patients already included in the study were excluded, as were the scans of patients known to have other malignancies. The data of the 50 patients in the fasting cohort were also used in a previous study (5). All patients gave written consent to the use of their anonymous data. Besides the standard ${ }^{18} \mathrm{~F}$-DCFPyL PET/CT examination, no additional measurements or actions affecting the patients were taken. The institutional review board approved this retrospective study, and the requirement to obtain informed consent was waived.

\section{Imaging}

${ }^{18} \mathrm{~F}$-DCFPyL was produced and synthesized by an on-site cyclotron facility. At 60 and $120 \mathrm{~min}$ after injection of ${ }^{18} \mathrm{~F}-\mathrm{DCFPyL}$, PET images were acquired on a Biograph-16 TruePoint PET/CT scanner (Siemens Healthcare). Images were acquired from the inguinal region to the base of the skull at $60 \mathrm{~min}$ after injection $(3 \mathrm{~min}$ per bed position) and from the inguinal region to the basal parts of the lungs at $120 \mathrm{~min}$ after injection ( $5 \mathrm{~min}$ per bed position).

Data were reconstructed using 3-dimensional iterative orderedsubsets expectation maximization, with 4 iterations, 16 subsets, and a 5 -mm gaussian filter. The image matrix was $256 \times 256$, pixel spacing was $2.67 \times 2.67 \mathrm{~mm}$, and slice thickness was $4 \mathrm{~mm}$. For attenuation correction of the 60-min PET images, radiocontrast-enhanced CT images were obtained (110 mAs at 110-130 kV), and for the 120-min PET images, low-dose CT images were obtained ( $25 \mathrm{mAs}$ at $110 \mathrm{kV})$. Collimation was $16 \times 1.2 \mathrm{~mm}$, pitch was 0.95 , slice thickness was $4 \mathrm{~mm}$, and the matrix was $512 \times 512$. The resulting voxel sizes were $1.37 \times 1.37 \mathrm{~mm}$ for the attenuation-correction CT images and $0.98 \times$ $0.98 \mathrm{~mm}$ for the diagnostic CT images.

\section{Lesion Selection and Data Extraction}

The images were read by 2 experienced nuclear medicine physicians. Activity $\left(\mathrm{SUV}_{\max }\right)$ in suspected lesions was measured by a volume-of-interest isocontour tool (40\% from maximum) using SyngoVia software (Siemens Healthcare), for a maximum of 4 lesions per 
anatomic location (prostate gland, seminal vesicles, local lymph nodes, distant lymph nodes, and bones). Lesions directly adjacent to tissues with more intense physiologic uptake were excluded from the analysis.

Activity was also measured in tissues known to express high physiologic uptake (submandibular gland, liver, spleen, duodenum, and other intestinal sections) using a standardized method (sphere volume of interest) $(2,3)$. Activity in the right submandibular gland was measured at only the first postinjection time point, whereas activity in the other tissues was measured at both time points. Other intestinal activity was measured, if present $\left(\mathrm{SUV}_{\max } \geq 3.0\right)$, in 4 different regions - up to $1 \mathrm{~cm}$ away from the left and right common iliac arteries and up to $1 \mathrm{~cm}$ away from the left and right external iliac arteries-since physiologic activity in those regions might interfere with detection of lymph node metastases.

\section{Statistical Analysis}

Normality of data was checked using the Kolmogorov-Smirnov test. Differences between cohorts or time points were tested using the independent-samples $t$ test, the Mann-Whitney $U$ test, the paired $t$ test, or the Wilcoxon signed-rank test. Bland-Altman plots were used for graphical presentation.

\section{RESULTS}

\section{Patients}

Two patients were excluded because they were scanned at deviant time points. In total, 50 and 48 patients were analyzed in the fasting and nonfasting cohorts, respectively (Table 1).

\section{Quantitative Analysis}

In the fasting and nonfasting cohorts, the respective number of lesions characteristic of prostate cancer totaled 152 and 131 (26 and 18 in the prostate gland, 5 and 5 in the seminal vesicles, 91 and 70 in lymph nodes, and 30 and 38 in bone). ${ }^{18} \mathrm{~F}$-DCFPyL uptake in those lesions did not differ between the cohorts at either time point (Table 2). Both cohorts showed a statistically significant increase in ${ }^{18} \mathrm{~F}$-DCFPyL uptake over time $(P<0.001$ for both, Wilcoxon

TABLE 1

Patient Characteristics

\begin{tabular}{lcc}
\hline \multicolumn{1}{c}{ Characteristic } & Fasting & Nonfasting \\
\hline Mean age $(\mathrm{y})$ & $73(52-84)$ & $71(53-83)$ \\
\hline Mean weight $(\mathrm{kg})$ & $83(44-112)$ & $86(64-118)$ \\
\hline Mean height $(\mathrm{m})$ & $1.78(1.60-1.96)$ & $1.79(1.68-1.97)$ \\
\hline $\begin{array}{c}\text { Mean body mass } \\
\left.\text { index (kg/m }{ }^{2}\right)\end{array}$ & $26(16-33)$ & $27(20-34)$ \\
\hline $\begin{array}{c}\text { Median dose }(\mathrm{MBq}) \\
\text { Median prostate- } \\
\text { specific antigen }\end{array}$ & $321(242-369)$ & $320(250-367)$ \\
\hline $\begin{array}{c}\text { Indication ( } n) \\
\text { Primary staging }\end{array}$ & $57.4(0.1-1,481)$ & $40.6(0.2-500)$ \\
\hline $\begin{array}{c}\text { Biochemical } \\
\text { recurrence }\end{array}$ & 16 & 11 \\
\hline $\begin{array}{c}\text { Follow-up of } \\
\text { systemic therapies }\end{array}$ & 22 & 33 \\
\hline $\begin{array}{c}\text { Other } \\
\text { Data in parentheses are range. }\end{array}$ & \\
\hline
\end{tabular}

signed-rank test) (Fig. 1). Separate analysis of the different anatomic locations showed that uptake in the prostate lesions was significantly higher in the fasting than the nonfasting cohort at both time points $(P=0.19$ at $60 \mathrm{~min}$ after injection [median, 17.7 and 10.9, respectively] and $P=0.26$ at $120 \mathrm{~min}$ after injection [median, 21.2 and 13.3, respectively], Mann-Whitney $U$ test). Uptake in nodal and distant metastases did not differ between the cohorts.

On quantitative measurement, normal ${ }^{18} \mathrm{~F}-\mathrm{DCFPyL}$ uptake was found in the submandibular gland for 50 and 47 fasting and nonfasting patients, respectively, in the liver for 50 and 48, in the spleen for 48 and 47, and in the duodenum for 50 and 48 . High activity was found in 38 other intestinal locations for 28 fasting patients and in 38 for 26 nonfasting patients. In the nonfasting cohort, uptake was significantly higher in the submandibular gland, which was imaged at 60 min after injection only, and in the liver and spleen at both time points but in the duodenum and other intestinal regions at only one time point (the duodenum at $60 \mathrm{~min}$ after injection [the earlier time point], the other intestinal

TABLE 2

Comparison of ${ }^{18} \mathrm{~F}-\mathrm{DCFPyL}$ Uptake

\begin{tabular}{|c|c|c|c|c|c|}
\hline Tissue & Cohort & $\begin{array}{l}\text { Minutes } \\
\text { after } \\
\text { injection }\end{array}$ & $n$ & SUV $_{\max }$ & $P$ \\
\hline \multirow[t]{4}{*}{ Malignant lesions } & Fasting & 60 & 152 & 10.2 & 0.420 \\
\hline & Nonfasting & & 131 & 10.5 & \\
\hline & Fasting & 120 & 152 & 12.5 & 0.391 \\
\hline & Nonfasting & & 131 & 12.8 & \\
\hline \multirow{4}{*}{$\begin{array}{l}\text { Submandibular } \\
\text { gland }^{*}\end{array}$} & Fasting & 60 & 50 & 16.2 & 0.017 \\
\hline & Nonfasting & & 47 & 18.7 & \\
\hline & Fasting & 120 & & & \\
\hline & Nonfasting & & & & \\
\hline \multirow[t]{4}{*}{ Liver } & Fasting & 60 & 50 & 6.8 & $<0.001$ \\
\hline & Nonfasting & & 48 & 7.7 & \\
\hline & Fasting & 120 & 50 & 6.9 & $<0.001$ \\
\hline & Nonfasting & & 48 & 7.9 & \\
\hline \multirow[t]{4}{*}{ Spleen } & Fasting & 60 & 48 & 5.6 & 0.035 \\
\hline & Nonfasting & & 47 & 6.4 & \\
\hline & Fasting & 120 & 48 & 4.6 & 0.050 \\
\hline & Nonfasting & & 47 & 4.8 & \\
\hline \multirow[t]{4}{*}{ Duodenum } & Fasting & 60 & 50 & 11.4 & 0.013 \\
\hline & Nonfasting & & 48 & 13.3 & \\
\hline & Fasting & 120 & 50 & 13.3 & 0.211 \\
\hline & Nonfasting & & 48 & 14.6 & \\
\hline \multirow[t]{4}{*}{ Intestine (other) } & Fasting & 60 & 38 & 4.3 & 0.116 \\
\hline & Nonfasting & & 38 & 4.9 & \\
\hline & Fasting & 120 & 38 & 3.8 & 0.006 \\
\hline & Nonfasting & & 38 & 5.5 & \\
\hline
\end{tabular}

*Activity in the right submandibular gland was measured at only the first postinjection time point, whereas activity in the other tissues was measured at both time points.

For malignant lesions, spleen, and intestine (other), SUV $\max$ is median and $P$ was obtained with Mann-Whitney $U$ test; for submandibular gland, liver, and duodenum, SUV $_{\max }$ is mean and $P$ was obtained with $t$ test. 


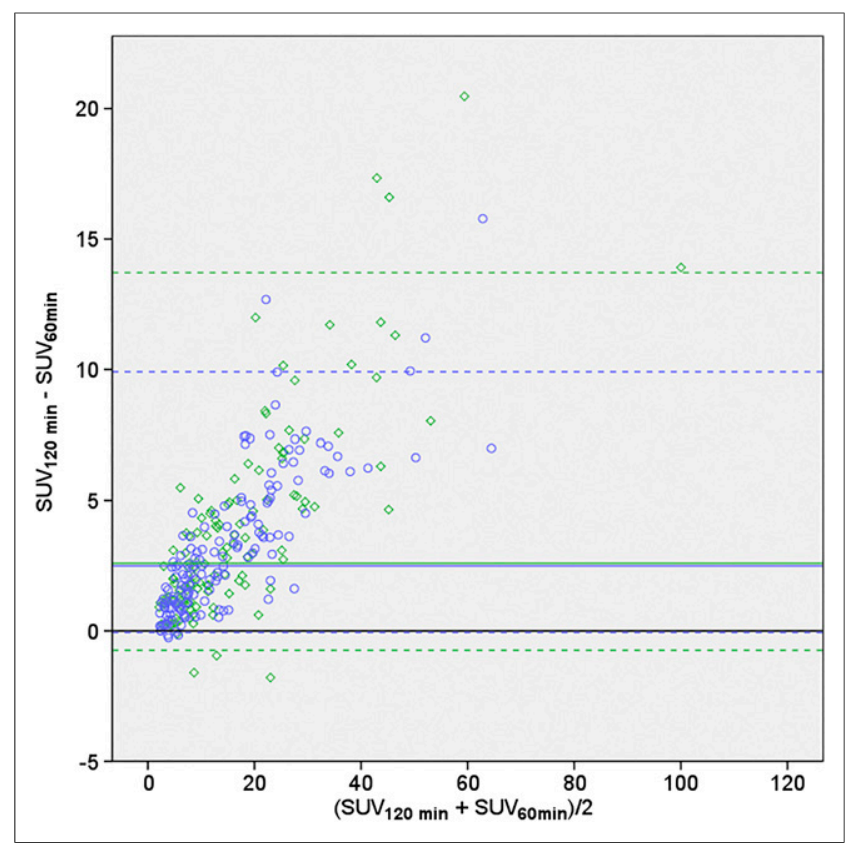

FIGURE 1. Difference between ${ }^{18} \mathrm{~F}-\mathrm{DCFPyL}$ activity at 120 and $60 \mathrm{~min}$ after injection plotted against mean activity in lesions characteristic of prostate cancer in fasting $(O)$ and nonfasting $(\diamond)$ cohorts. regions at $120 \mathrm{~min}$ after injection [the later time point]) (Table 2). Activity in the spleen decreased significantly over time in both cohorts $(P<0.001$ for both, Wilcoxon signed-rank test). Uptake in the liver, duodenum, and other intestinal regions showed a variable course over time on a per-patient basis (Fig. 2); however, a significant increase in $\mathrm{SUV}_{\text {max }}$ over time was found in the duodenum $(P<0.001$ and $P=0.001$ in the fasting and nonfasting cohorts, respectively; paired $t$ test).

\section{Validation of Suspected Lesions}

Of 44 suspected prostate lesions, 31 were histopathologically proven to be malignant and 5 showed indirect signs of malignancy (decreased prostate-specific antigen levels after therapy or confirmation by other imaging procedures). For 121 of 161 suspected lymph nodes and 66 of 68 suspected bone lesions, indirect signs of malignancy were present (confirmation by other imaging procedures or further imaging during follow-up). Other or further imaging procedures comprised CT, MRI, ${ }^{99 m}$ Tc-hydroxydiphosphonate bone scintigraphy, ${ }^{18} \mathrm{~F}$-sodiumfluoride PET/CT, or ${ }^{18} \mathrm{~F}$ - or ${ }^{68} \mathrm{Ga}$-PSMA PET/CT.

\section{DISCUSSION}

In general, lesions characteristic of prostate cancer showed similar uptake in both the fasting and the nonfasting cohorts. Physiologic uptake was higher in the nonfasting cohort, but the clinical relevance of this finding is questionable. Prostate cancer metastasizes to the lymph nodes and skeleton before involving other organs (6). There-

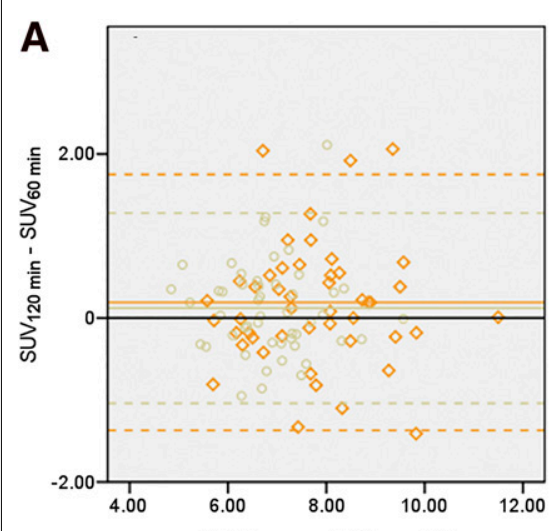

$\left(\mathrm{SUV}_{120 \mathrm{~min}}-\mathrm{SUV}_{60 \mathrm{~min}}\right) / 2$

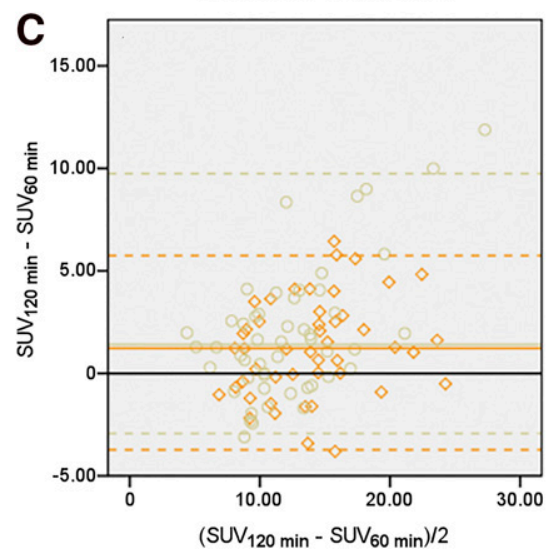

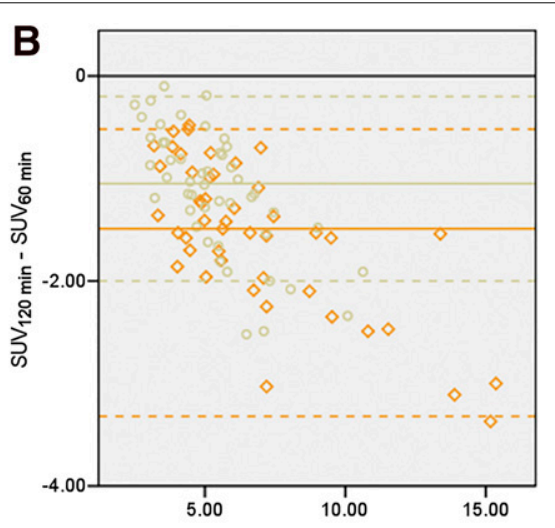

$\left(\mathrm{SUV}_{120 \mathrm{~min}}+\mathrm{SUV}_{60 \mathrm{~min}}\right) / 2$

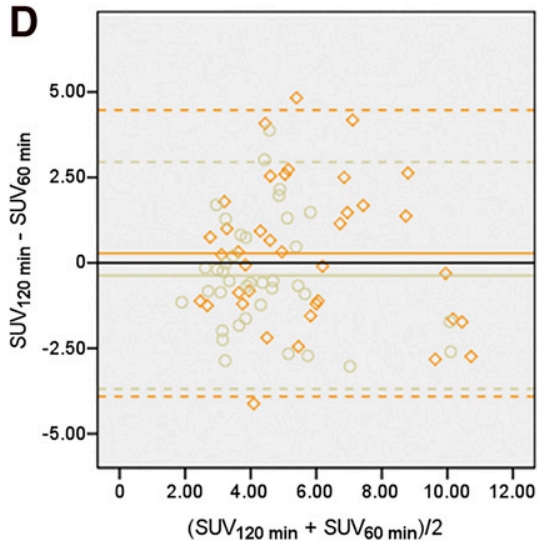

FIGURE 2. Difference between ${ }^{18} \mathrm{~F}$-DCFPyL activity at 120 and 60 min after injection plotted against mean activity in liver (A), spleen (B), duodenum (C), and other intestinal locations (D) in fasting $(\circ)$ and nonfasting $(\diamond)$ cohorts. fore, the presence of metastasis in other organs usually has limited consequences for patient management. Metastasis detection can be hampered by high physiologic uptake in structures directly adjacent to metastases.

For lymph node metastases, which occur predominantly in the pelvis and abdomen, detection may be hampered by activity in the aortic and iliac blood pool, in surrounding adipose tissue, in adjacent intestine (Fig. 3), and in the ureters and bladder. Activity in the blood pool and adipose tissue is generally low $(2,5)$. The frequency of intestinal activity was similar between the cohorts. Urinary activity in the ureters and bladder is largely influenced by the level of hydration (use of diuretics and voiding) rather than by fasting. Activity in bone marrow and in muscle adjacent to bone may interfere with detection of bone metastases. However, physiologic activity in these tissues is generally low $(2,5)$.

The present data do not elucidate the cause of higher physiologic ${ }^{18} \mathrm{~F}-\mathrm{DCFPyL}$ uptake in the nonfasting cohort. It is questionable whether ${ }^{18} \mathrm{~F}$-DCFPyL activity in most organs is very specific. PSMA ligands have affinity for the enzyme glutamatecarboxypeptidase II, but there is no reason to assume that the activity of this enzyme increases after food intake.

The surprising difference in the $\mathrm{SUV}_{\max }$ of prostate lesions between cohorts was 


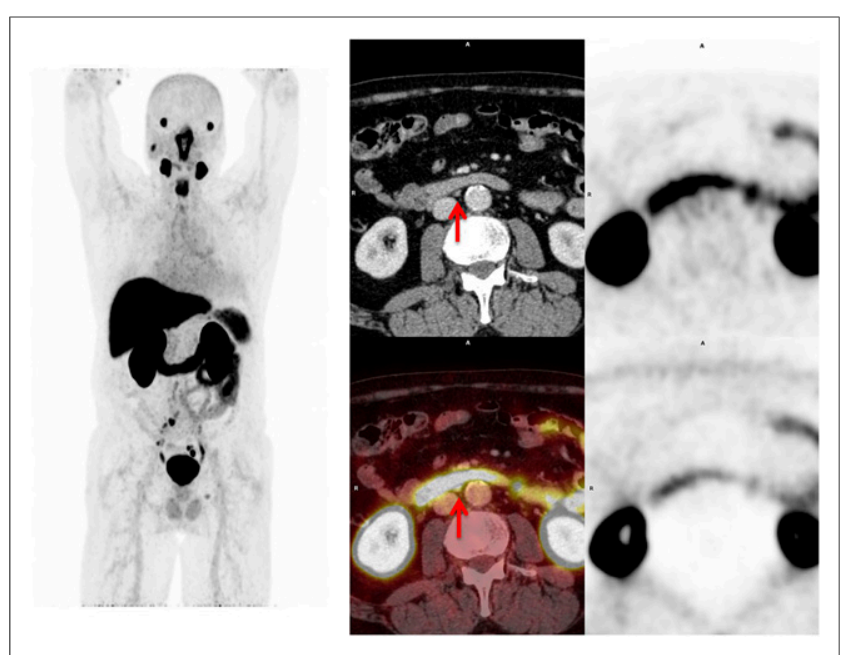

FIGURE 3. Interference of physiologic ${ }^{18} \mathrm{~F}-\mathrm{DCFPyL}$ uptake in duodenum with detection of potential lymph node metastasis (arrows) on maximumintensity projection (left), axial CT and fused ${ }^{18} \mathrm{~F}$-DCFPyL PET/CT (middle), and axial AC and NAC ${ }^{18}$ F-DCFPyL PET (right) images.

probably caused by other differences between the cohorts. The primary tumor was still in situ in $81 \%$ of patients in the fasting cohort but in only $61 \%$ of patients in the nonfasting cohort. Uptake is typically much higher in primary tumors than in the relatively small local biochemical recurrences.

The effects of fasting may become clinically relevant in patients who are treated with ${ }^{177}$ Lu-PSMA-617 or ${ }^{225}$ Ac-PSMA-617. Dosimetry studies have shown that the salivary glands and kidneys might be dose-limiting organs for ${ }^{177} \mathrm{Lu}$-PSMA-617 (7). From this perspective, the effects of fasting on kidney activity are also interesting; however, the patients in our study were using various diuretic regimens, which would affect ${ }^{18} \mathrm{~F}$-DCFPyL uptake in the kidneys. Therefore, kidney activity was not included in the analysis. For ${ }^{225}$ Ac-PSMA-617, it has been empirically found that severe xerostomia is the dose-limiting toxicity (8). According to our data, fasting resulted in $13 \%$ less ${ }^{18} \mathrm{~F}-\mathrm{DCFPyL}$ uptake in the submandibular glands at $60 \mathrm{~min}$ after injection. It may be of interest to study the effects of fasting during PSMA therapy.

A potential limitation of our study was that attenuationcorrection CT was performed with radiocontrast enhancement for the 60-min PET images but not for the 120-min PET images. No literature can be found on the effects of enhanced CT on SUV measurements in ${ }^{18} \mathrm{~F}$-DCFPyL PET/CT. However, in ${ }^{18} \mathrm{~F}-\mathrm{FDG}$ PET/CT, studies on this topic have shown enhanced CT to have little to no clinical impact on SUV measurements (9). The advantages of enhanced CT for characterization of lymph nodes adjacent to vascular structures or ureters outweighed any potential interference with SUV measurements. SUV may be slightly higher during the earlier phase than later, but this effect would equally apply to both cohorts. Lesions that were characteristic of prostate cancer showed a significant increase in $\mathrm{SUV}_{\max }$ over time. If radiocontrast affected SUV measurements, the difference would have been even higher than that observed.

Another potential limitation was lack of the gold standard, histopathologic confirmation, for reference. From a practical and ethical standpoint, histopathologic confirmation of all lesions in this study was not possible. However, most lesions showed indirect signs of malignancy. Furthermore, because ${ }^{68} \mathrm{Ga}$-labeled PSMA tracers are highly specific for prostate cancer, prostate cancer should be highly suspected in lesions that have increased ${ }^{18}$ F-DCFPyL uptake and fit the pattern of metastatic spread of prostate cancer (10).

\section{CONCLUSION}

According to our data, fasting for up to $6 \mathrm{~h}$ before ${ }^{18} \mathrm{~F}-\mathrm{DCFPyL}$ PET/CT does not significantly affect uptake in suspected malignant lesions but significantly lowers uptake in tissues with high physiologic uptake, such as the salivary glands, liver, and spleen. The absolute differences in ${ }^{18} \mathrm{~F}-\mathrm{DCFPyL}$ uptake are relatively small, and any effects on diagnostic performance can therefore be considered negligible.

\section{DISCLOSURE}

No potential conflict of interest relevant to this article was reported.

\section{REFERENCES}

1. Bluemel C, Krebs M, Polat B, et al. ${ }^{68} \mathrm{Ga}$-PSMA-PET/CT in patients with biochemical prostate cancer recurrence and negative ${ }^{18} \mathrm{~F}$-choline-PET/CT. Clin Nucl Med. 2016;41:515-521.

2. Szabo Z, Mena E, Rowe SP, et al. Initial evaluation of $\left[{ }^{18} \mathrm{~F}\right] \mathrm{DCFPyL}$ for prostatespecific membrane antigen (PSMA)-targeted PET imaging of prostate cancer. Mol Imaging Biol. 2015;17:565-574.

3. Li X, Rowe SP, Leal JP, et al. Semiquantitative parameters in PSMA-targeted PET imaging with ${ }^{18} \mathrm{~F}$-DCFPyL: variability in normal-organ uptake. J Nucl Med. 2017;58:942-946.

4. Fendler WP, Eiber M, Beheshti M, et al. ${ }^{68} \mathrm{Ga}$-PSMA PET/CT: joint EANM and SNMMI procedure guideline for prostate cancer imaging-version 1.0. Eur $J$ Nucl Med Mol Imaging. 2017;44:1014-1024.

5. Wondergem M, van der Zant FM, Knol RJJ, Lazarenko SV, Pruim J, de Jong IJ. ${ }^{18} \mathrm{~F}$-DCFPyL PET/CT in the detection of prostate cancer at 60 and 120 minutes: detection rate, image quality, activity kinetics, and biodistribution. J Nucl Med. 2017;58:1797-1804.

6. Bubendorf L, Schopfer A, Wagner U, et al. Metastatic patterns of prostate cancer: an autopsy study of 1,589 patients. Hum Pathol. 2000;31:578-583.

7. Kabasakal L, AbuQbeitah M, Aygun A, et al. Pre-therapeutic dosimetry of normal organs and tissues of ${ }^{177}$ Lu-PSMA-617 prostate-specific membrane antigen (PSMA) inhibitor in patients with castration-resistant prostate cancer. Eur J Nucl Med Mol Imaging. 2015;42:1976-1983.

8. Kratochwil C, Bruchertseifer F, Rathke H, et al. Targeted alpha-therapy of metastatic castration-resistant prostate cancer with ${ }^{225}$ Ac-PSMA-617: dosimetry estimate and empiric dose finding. J Nucl Med. 2017;58:1624-1631.

9. Mawlawi O, Erasmus JJ, Munden RF, et al. Quantifying the effect of IV contrast media on integrated PET/CT: clinical evaluation. AJR. 2006;186:308-319.

10. Rauscher I, Maurer T, Beer AJ, et al. Value of ${ }^{68}$ Ga-PSMA HBED-CC PET for the assessment of lymph node metastases in prostate cancer patients with biochemical recurrence: comparison with histopathology after salvage lymphadenectomy. J Nucl Med. 2016;57:1713-1719. 